\title{
Factores que Influyen en la Erosión Hídrica del Complejo Arqueológico de Vilcashuamán, Perú 2021
}

\section{Factors that Influence the Water Erosion of the Vilcashuamán Archaeological Complex, Peru 2021}

\begin{abstract}
Rosmery Salinas-Ayala
Universidad Nacional Autónoma de Huanta, Ayacucho, Perú E-mail: 1712820118@unah.edu.pe ORCID: https://orcid.org/0000-0002-6146-2140

Kevin Sánchez-Magallanes

Universidad Nacional de Cañete, Lima Perú E-mail: kevinsanmaga12@gmail.com ORCID: https://orcid.org/0000-0002-2134-9346

Carlos Pozo-Curo Universidad Nacional Autónoma de Huanta, Ayacucho, Perú Email: cpozo@unah.edu.pe ORCID: https://orcid.org/0000-0003-1464-335X

Elvis Doberto Osorio-Arrascue Universidad San Martin de Porres, Perú Email: eosorioarrascue@gmail.com ORCID: https://orcid.org/0000-0003-1395-7242
\end{abstract}

\section{Resumen}

Está investigación tiene como objetivo identificar los factores que influyen en la erosión del complejo Arqueológico de Vilcashuamán, por los siguientes factores: Iluvias, el calor, algunas maniobras incorrectas, no respetar el aforo y el mal cuidado del complejo los cuales, ocasionan el desgaste de las piedras generando hongos en la planta superior, así mismo se planteó una solución para el drenaje del agua las cuales son causales de la erosión, así mismo ordenar las visitas realizadas para una buena satisfacción de los turistas y de la población misma generando un ingreso económico para su familia e involucrarse más en el sector turismo, el cuidado y el buen marketeo ayudara a una visita fluida y es lo que se quiere lograr, la investigación tuvo enfoque cualitativo, tipo descriptivo. Los resultados indicaron que existen 4 grupos de edades que visitan el Complejo Arqueológico de 
Vilcashuamán, siendo el $36.7 \%$ los visitantes más recurrentes. Finalmente, se concluye que los posibles factores de la erosión en el sitio estudiado podrían ser el tiempo climatológico, aforo máximo de visitantes y el tiempo que estos residen.

\title{
Palabras Clave
}

Erosión hídrica, complejo arqueológico, afluencia turística.

\begin{abstract}
The objective of the investigation is to identify the Factors that influence the erosion of the Vilcashuamán Archaeological complex due to the following factors: rain, heat, some incorrect maneuvers, not respecting the capacity and poor care of the complex as this causes the wear of the stones generating fungi on the upper floor, likewise, a solution for the drainage of water will be proposed, which are causes of erosion, as well as ordering the visits made for a good satisfaction of tourists and the population itself, having an economic income for their family and get more involved in the tourism sector, care and good marketing will help a smooth visit and is what you want to achieve. The research had a qualitative, descriptive approach. The results indicated that there are 4 age groups that visit the Vilcashuamán Archaeological Complex, with $36.7 \%$ being the most frequent visitors. Finally, it is concluded that the possible factors of erosion in the studied site could be the climatological weather, maximum capacity of visitors and the time they reside.
\end{abstract}

\section{Keywords}

Water erosion, Archaeological complex, tourist afluence.

\section{Resumo}

Esta pesquisa tem como objetivo identificar os fatores que influenciam a erosão do complexo arqueológico de Vilcas Huamán pelos seguintes fatores: chuva, calor, algumas manobras incorretas, não respeitando a aferição e o mau cuidado do complexo, pois isto causa o desgaste das pedras gerando fungos no piso superior, A pesquisa teve uma abordagem qualitativa, de tipo descritivo. Os resultados indicam que há 4 grupos etários que visitam o Complexo Arqueológico de Vilcashuamán, sendo 36,7\% os visitantes mais freqüentes. Finalmente, conclui-se que os possíveis fatores de erosão no local estudado poderiam ser o clima climatológico, a capacidade máxima dos visitantes e o tempo em que residem.

\section{Palavras-chave}

Erosão hídrica, complexo arqueológico, afluência turística. 


\section{Introducción}

La erosión hídrica es un fenómeno que es entendida como la remoción, transporte y la deposición de las partículas de suelo, materia orgánica y se presentan con distintos grados de intensidad puede ser uno mayor que el otro dependiendo del lugar donde se encuentra la temperatura así mismo el mismo tiempo de lugar (Sabino et al., 2017). Vilcashuamán es un distrito que se encuentra en 3,490 msnm aproximadamente y con esta altitud es fácil que se encuentre en una degradación mayor que a los Delmas, así mismo el aforo de las personas al ingresar al complejo arqueológico de Vilcashuamán también hace que la erosión de este complejo se acelere de tal manera que podría ser imposible de restaurar y esto podría provocar el desequilibrio de la estabilidad de las paredes del complejo y los demás sitios arquitectónicos dentro del distrito .

La erosión de los suelos es algo dinámico, es un proceso de degradación que se dan perdiendo la fertilidad de ella con las constantes lluvias, el calor, la humedad, los hongos que se depositan en las piedras al no estar adecuadamente cuidadas, la otra causa es por el ingreso de las personas visitantes excediendo el limite de aforo establecido que no ayudan en el cuidado de las piedras.

Según (Zaccagnini et al., 2014) en la Argentina se realiza un acueducto de sistematización de terrazas para el control de la erosión hídrica y aguas superficiales, para ello plantean que se debe construir un paño que tenga la cualidad de una bajada para llegar al canal y después de ello se realiza un lomo para que el agua ingrese al canal sin llegar que esta se desvíe. De esta misma manera, en el complejo arqueológico de Vilcashuamán se puede construir este tipo de drenajes teniendo todos los cuidados para que el agua causada por las lluvias no llegue a empozarse en el lugar, sino, más bien esta sea absorbida por el canal. Para ello, se debería realizar un estudio de la zona con la finalidad de realizar estratégicamente la ubicación del paño, el canal y el lomo.

Un tema con similar situación fue trabajado en la ciudad de Trujillo y que trata sobre los factores Meteorológicos, geofísicos que influyen en el proceso de erosión de las 
superficies arquitectónicas. Dentro de ello, se menciona la manera de trabajar para una restauración del caso de la Huaca de la Luna en el valle Moche.

Los monumentos arqueológicos de tierra en la costa del norte de Perú, considerado como una herencia cultural, protegida o no protegida se encuentra en un estado crítico y ello va en desmedro de su integridad físico-mecánica y de su potencial turístico (Morales, 2011).

\section{Estado del arte o Marco Teórico}

Esta sección muestra un recorrido de enfoques teóricos de autores que han investigado el tema o fenómeno de la investigación.

\subsection{Erosión Hídrica}

Es un fenómeno diversificado en sus modalidades (Sala, 1984). Esta consiste en la remoción del suelo como partículas, así mismo se señala que estos pueden tener distintos factores de deterioro como el clima, sismos y, estas provocan la erosión del suelo con el desequilibrio de la estabilidad de la tierra o de un complejo (SENAMHI, 2017).

La erosión es un proceso de desprendimiento del suelo o la superficie, así mismo ocurre en el transporte de zonas que fueron removidas, la erosión ocurre naturalmente de la misma manera esta puede ser en efecto del agua sobre el suelo (Zaccagnini et al., 2014).

La Institución (SENAMHI, 2017) de la misma manera señala que un proceso de la erosión, como la primera fase tiene el transporte. Esta consiste en la separación y el desplazamiento de los materiales desgastados. Como segunda fase, la acumulación que al encontrarse en una pendiente se llegará a dar el desplazamiento y la sedimentación de esta.

De la misma manera (Morales, 2011) nos habla de otro tipo de erosión que puede influir dentro de un complejo como el caso Huacas de Moche, Valle Moche haciendo un estudio matemático concluye que se puede dar una erosión eólica y la contaminación de los monumentos arqueológicos esta puede tener un deterioro de mayor impacto en las superficies arquitectónicas. 


\subsection{Complejo Arqueológico}

Cuando se trata de complejos arqueológicos, se debe realizar un monitoreo permanente y realizar las características de los factores de deterioro que pueda definir la patología del complejo Arqueológico, así mismo el diseño de la preservación y conservación del monumento (Morales, 2011).

Según el (Ministerio de Cultura, 2014), los monumentos arqueológicos, son construcciones prehispánicas, que dan evidencia a la actividad de nuestros antepasados, entonces si se aplica al Perú (Vilcashuamán) pertenecería a este rubro, no obstante, si se trata de un edificio aislado o de un "complejo Arqueológico o una zona arqueológica monumental" es decir un conjunto de edificios (Espinoza-Pajuelo, 2016).

Entonces podemos mencionar que en Vilcashuamán existe un conjunto de edificaciones talladas como el Ushno, el Templo del Sol, la Plaza, entre otros y es considerado como el Complejo Arqueológico de Vilcashuamán.

\subsection{Afluencia Turística}

Según el (Destino Negocio, 2015) el Ministerio de Comercio Exterior y Turismo afirma que la afluencia turística en el Perú fue en aumento año tras año pero para el 2015 el número de turistas incrementó en un $8.4 \%$ que hace referente a más de 2 millones de visitantes que ingresaron a nuestro país, esto evidencia una mayor afluencia turística en las regiones, atractivos turísticos y complejos arqueológicos que trae beneficios económicos, empero, por el lado negativo se tiene el aumento de aforo en los lugares turísticos y puede ser una de las tantas causas para la erosión del lugar.

\section{Materiales y métodos}

El presente estudio corresponde al enfoque cuantitativo, tipo descriptivo, diseño no experimental, de corte transversal. La población estuvo conformada por Guías oficial de turismo, población de Vilcashuamán de Ayacucho - Perú; la muestra estuvo representada por 30 visitantes, Guías de turismo, población de Vilcashuamán de Ayacucho, siendo el 
muestreo no probabilístico intencional, la técnica utilizada fue la encuesta online. Cada encuesta consta de 10 preguntas.

\section{Resultados}

En la figura 1 según la encuesta a 30 personas y la pregunta sobre edades nos dio un resultado que un $36.7 \%$ tienen de 25 a 37 años seguidamente tenemos a las personas de 35 a 44 años con un $26.7 \%$. Estas serían las edades de las personas encuestadas que visitaron el Complejo Arqueológico de Vilcashuamán.

\section{Figura 1}

Edad - 30 respuestas

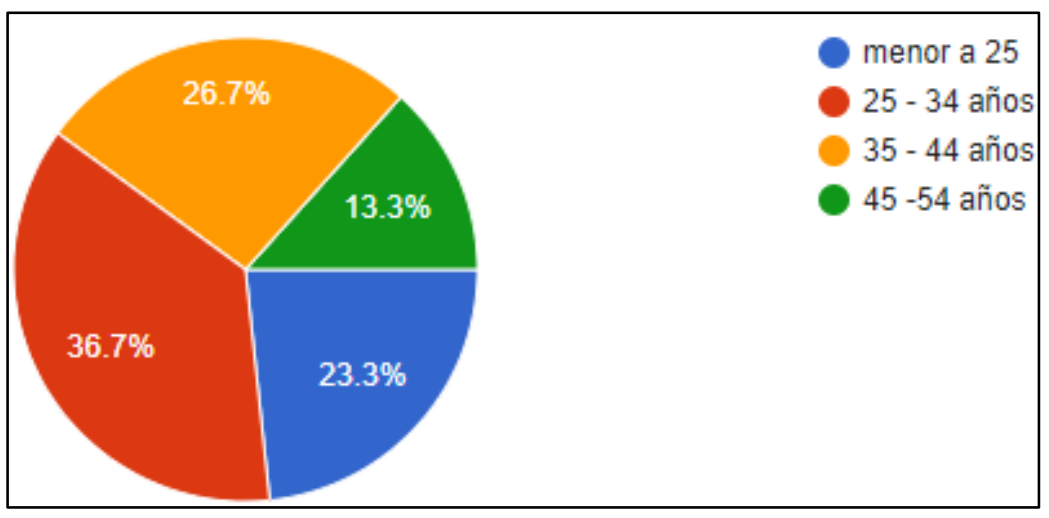

Fuente: Elaboración Propia

En cuanto a la Figura 2 ¿Cuántas veces visitó el Complejo Arqueológico de Vilcashuamán? La respuesta fue el siguiente, considerando que esta encuesta se hizo a Guías y visitantes a la zona, se obtuvo que un $46.7 \%$ visitaron de 1 a 3 veces por año, seguidamente de un $36.7 \%$ visitaron por lo menos 1 a 3 veces por mes y un $16.7 \%$ visitó de 1 a 3 veces por semana, eso quiere decir que se registró una buena cantidad de personas que vistan el complejo Arqueológico de Vilcashuamán.

\section{Figura 2}

¿Cuántas veces visitó el Complejo Arqueológico de Vilcashuamán? 


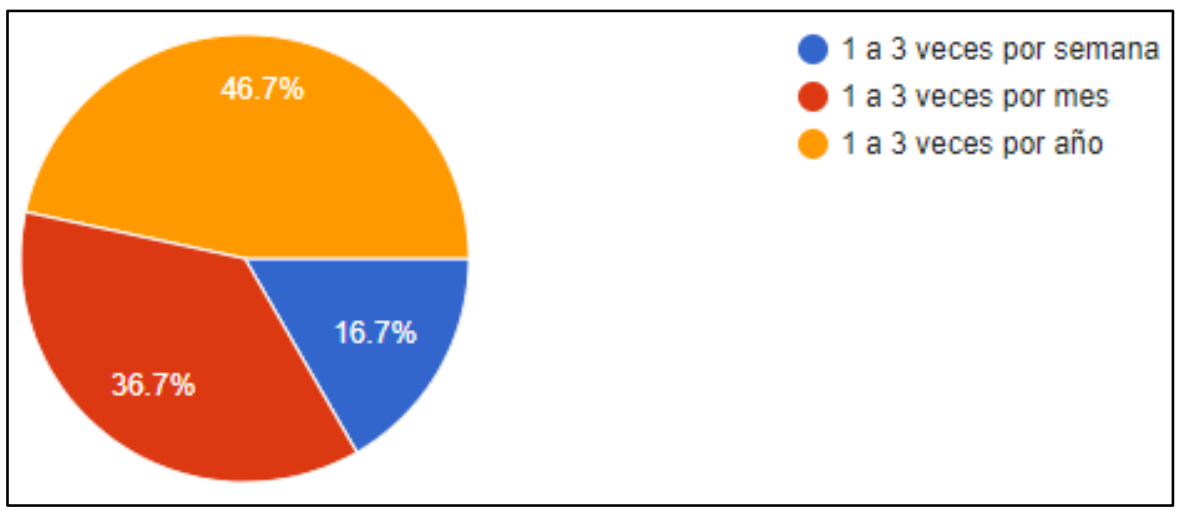

Fuente: Elaboración Propia

En la figura 3, ¿Durante su visita observó alguna persona indicando las zonas que no se debe acceder? las respuestas en su mayoría representaron el $80 \%$ de las personas encuestadas indicaron que no observan personal alguno, mientras que un $20 \%$ indicaron que si observaron a personas indicando que hay zonas a las cuales no se puede acceder. En conclusión, se puede mencionar que, en su mayoría de los días de visitas, no hay un personal que informe el no acceso a zonas prohibidas, dando libertad de acceso sin control.

\section{Figura 3}

¿Durante su visita observó alguna persona indicando las zonas que no se debe acceder?

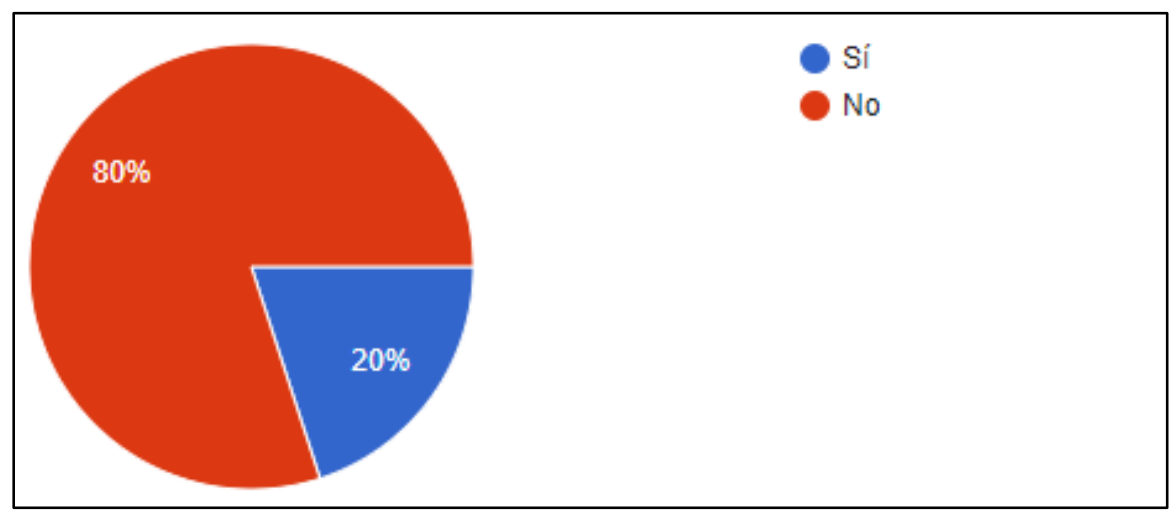

Fuente: Elaboración Propia

\section{Discusión}

En la figura 1, se muestra que a través de los resultados de la encuesta se determina que existen 4 rangos de edades que visitaron el Complejo Arqueológico de Vilcashuamán, 
el $36.7 \%$ corresponde de 25 a 34 años, $26.7 \%$ de 35 a 44 años, $23.3 \%$ de 1 a 25 años y $13.3 \%$ de 45 a 54 años. Esto nos hace reflexionar un caso similar con la investigación de (Vázquez \& Vázquez, 2017) en la cual, realizan el aprovechamiento turístico de la riqueza material y cultural de tres municipios en Potosí, Bolivia. Conformando un importante corredor turístico con los atributos más importantes del lugar.

En la figura 2, la encuesta permitió determinar la cantidad de visitas anual, mensual y semanal, obteniendo que el 46.7\% visitaron de 1 a 3 veces por año, 36.7\% visitaron de 1 a 3 veces por mes y el 16.7\% visitaron de 1 a 3 veces por semana al Complejo Arqueológico de Vilcashuamán. Lo cual coincide con las investigaciones de (Ignacio, 2017) que concluye que se debe promover la expansión del turismo como una estrategia de desarrollo económico local sobre todo en las zonas caracterizadas por alta marginalidad y fragilidad socio ambiental.

En la figura 3, se observa que la encuesta permitió determinar que el $80 \%$ de los visitantes indican que no se dieron cuenta de algún personal brindado información o guía en zonas prohibidas y el $20 \%$ indicaron que si pudieron notar la presencia de personal que indican el acceso o restricción a las zonas disponibles para la visita arqueológica. Lo cual coincide con (Muñoz, 2017) que afirmó que los residentes de un lugar turístico en Ecuador aprovechan el turismo de la zona para sacar el máximo provecho sin importar los impactos, realizan actividades informales, alojamientos en casas sin registro, taxistas que ofrecen tours sin permiso y guías de turismo sin licencia.

\section{Conclusiones}

De acuerdo a la investigación realizada podemos afirmar que los posibles factores de la erosión en el complejo arqueológico de Vilcashuamán son por el tiempo y los fenómenos naturales como la lluvia y calor adicionalmente al otro factor que viene a ser el exceso en el aforo máximo de visitantes que puede recibir el complejo, teniendo en cuenta el incremento de visitar en las fiestas festivas. Así mismo, el factor de tiempo de permanencia de las personas que se quedan en los complejos arqueológicos puede generar que haya un desgaste fácil de las piedras, paredes y suelo del complejo. De igual manera, el ingreso de algunas personas que acceden a algunas zonas que no deberían acceder, por ejemplo, según el comentario de una Guía de turismo no hay un mantenimiento de los hongos que hay en las piedras entre otros, en consecuencia, se encuentra en un mal estado. 
En ese sentido, se debe efectuar un tratamiento especial contra los hongos de las piedras arqueológicas y la implementación con la construcción de un canal conductor de agua hacia una canaleta y no se quede estancada como un charco dentro del complejo, cuyo estancamiento de agua en charcos, pueda generar deterioros de las piedras arqueológicas. Ello, ayudaría a que este complejo cuente con mantenimiento permanente sin que esta se desplome y, en consecuencia, contar con mayor frecuencia de visitas alargando el tiempo de vida útil del complejo. Así mismo, es necesario la ayuda de todos citadinos, por un lado, concienciando y teniendo una persona encargada dentro del complejo para brindar información sobre la situación que se encuentra los restos arqueológicos y las restricciones de acceso a zonas en deterioro o de mayor cuidado.

\section{Referencias}

Espinoza-Pajuelo, P. D. (2016). La gestión de monumentos arqueológicos en Lima, Perú: panorama, diagnóstico y propuesta. Córima, Revista De Investigación En Gestión Cultural (e-ISSN 2448-7694), 2(2). https://doi.org/10.32870/cor.a2n2.6083

Ignacio, C. (2017). Un destino incierto. Expansión del turismo, daños y riesgos ambientales en la costa de Oaxaca. Acta Sociológica, 73, 83-122.

https://doi.org/10.1016/J.ACSO.2017.08.003

Destino Negocio. (2015). Crece el número de turistas que llegan al Perú.

https://destinonegocio.com/pe/economia-pe/crece-el-numero-de-turistas-quellegan-al-peru/

Ministerio de Cultura. (2014). Reglamento de Intervenciones Arqueológicas. Normas Legales del 04.10.2014.

https://repositorio.cultura.gob.pe/bitstream/handle/CULTURA/605/ds003-2014-mcria.pdf?sequence $=1 \&$ isAllowed $=y$

Morales, R. (2011). Factores meteorológicos, geofísicos y químicos que influyen el proceso de erosión de las superficies arquitectónicas de tierra: caso huaca de la Luna, valle Moche, Trujillo. 
Muñoz, A. (2017). Percepciones de la gestión del turismo en dos reservas de biosfera ecuatorianas: Galápagos y Sumaco. Investigaciones Geográficas, Boletín Del Instituto de Geografía, 2017(93), 110-125. https://doi.org/10.14350/RIG.47805

Sabino, E., Felipe, O., \& Lavado, W. (2017). Atlas de erosión de suelos por regiones hidrológicas del Perú. Nota Técnica Nº02 SENAMHI-DHI-2017.

Sala, M. (1984). Geomorfología actual. Guía conceptual, temática y bibliográfica. Revista de Geografia, 209-248.

SENAMHI. (2017). Atlas de Erosión de Suelos por Regiones Hidrológicas del Perú. Nota tecnica 002 -SENAMHI-DHI-2017. https://idesep.senamhi.gob.pe/portalidesep/files/tematica/atlas/erosi\%C3\%B3n_de_ suelo/Atlas_Erosion_Suelos_por_Regiones_Hidrologicas_Peru.pdf

Vázquez, G., \& Vázquez, V. (2017). Evaluación de recursos naturales y culturales para la creación de un corredor turístico en el altiplano de San Luis Potosí, México. Investigaciones Geográficas, Boletín Del Instituto de Geografía, 2017(94), 91-105. https://doi.org/10.14350/RIG.56575

Zaccagnini, M., Wilson, M., Calamari, N., Goijman, A., Solari, L., Suarez, R., Decarre, J., Gavier, G., Gabioud, E., Sasal, M., Stamatti, G., Dardanelli. Sebastián, Oszust, J., \& Revora, S. (2014, August). Manual de buenas prácticas para la conservación del suelo, la biodiversidad y sus servicios ecosistémicos. 